\title{
Adaptive Control of the Methane Flow Rate in Biogas Plants
}

\author{
Khadidja Chaib Draa \\ Automatic Control Research Group \\ University of Luxembourg, Luxembourg \\ khadidja.chaibdraa@uni.lu
}

\author{
Holger Voos \\ Automatic Control Research Group \\ University of Luxembourg, Luxembourg \\ holger.voos@uni.lu
}

\begin{abstract}
In this paper we consider the control of the methane flow rate in biogas production using both a continuous stirred tank reactor and a fixed bed digester. The goal is to regulate the methane flow rate in order to match an energy demand in spite of variations in the waste concentrations. For this purpose, a two step (acidogenesis-methanogenesis) nonlinear mass balance model is considered. Due to the costs and complexity of the measurements an adaptive observer is designed for the acidogenesis reaction rate. The stability of the observation part is proved and its implementation with the designed adaptive control law for the methane flow rate and the acetate concentration in the reactor are confirmed by simulations.

Index Terms-Anaerobic Digestion; Linearized Control; Adaptive Observer; Nonlinear Models.
\end{abstract}

\section{INTRODUCTION}

The Anaerobic Digestion (AD) is a hopeful process for the production of biogas and thus supporting waste recovery and protection of the environment. It comprises a transformation of organic matter (proteins, fats and carbohydrates) involving several chemical and physicochemical reactions in series and/or in parallel, mediated by anaerobic bacteria, into biogas. The latter can be considered as a renewable energy source which could be either cleaned and upgraded to natural gas standards or directly used in gas engines to be converted into electricity and heat. One general idea of our research is the integration of biogas plants (BPs) in the concept of virtual power plants (VPP). A VPP comprises a larger set of electric power sources with fluctuating single amounts of generated power (such as wind turbines or photovoltaic plants) which are controlled and coordinated in a way that the overall generated power mimics the characteristics of one single large power plant. In order to integrate biogas plants in a most efficient way into VPPs, our focus is on the control of the generated methane gas flow rate in spite of fluctuating amounts of incoming waste material and thus turning the gas flow rate into an additional controllable input variable for the overall coordination process of the VPP.

However, controlling the AD process in the biogas plant is a difficult task since it involves living organisms which are very sensitive to the operating conditions and may be inhibited or washed out in the worst case leading to a definite stop of the digester. Furthermore, the power demand satisfaction should not be at the expense of destabilizing the biologic AD process. Therefore, a total fulfilment of any power demand is not possible if this results in an unsafe operating mode of the digester and hence the methane gas flow rate will be constrained by the safe operating conditions of the digester. In addition, in order to control a complex process like the $\mathrm{AD}$ a suitable model giving an a priori knowledge of the process is needed first to design a control law.

Therefore we start here in Section II with the brief derivation of a model which provides a compromise between modelling accuracy and model complexity with respect to a model-based controller design. Then, in Section III we design a linearized controller for the methane gas flow rate, followed by the design of a control law for the acetate concentration inside the reactor as presented in Section IV. Moreover, since the full vector of state variables is not measurable we build an observerbased estimator for the acidogenesis reaction rate and prove its stability in Section V. Finally, in Section VI we give the full algorithm ensuring the digester's safety while controlling the methane flow rate. Before concluding the paper and giving some future work perspectives in Section VIII, we evaluate the proposed algorithm by simulations in Section VII where the influent concentrations are fluctuating.

\section{MODEL DeSCRIPTION}

In the literature we can find more than 70 dynamic models of the AD process, see e.g. [1] and [2] for an overview. Most of these models are complex and not suitable for control design because they focus on the modelling of all detailed aspects of the biological process, such as for instance the anaerobic digestion model No.1 (ADM1) [3] which contains 32 differential equations. However, all models have in common that they consider the Volatile Fatty Acids (VFA) as limiting intermediates which can inhibit the methanogenic bacteria by their accumulation. Therefore, it has been reported in [5], [6], [7], and [9] that a two-step (acidogenesis and methanogenesis) model is sufficient for the AD analysis and control. These two important steps are described by the following chemical reactions:

1) Acidogenesis with reaction rate $r_{1}=\mu_{1} X_{1}$ :

$$
k_{1} S_{1} \stackrel{r_{1}}{\rightarrow} X_{1}+k_{2} S_{2}+k_{4} C O_{2}
$$

2) Methanogenesis with reaction rate $r_{2}=\mu_{2} X_{2}$ :

$$
k_{3} S_{2} \stackrel{r_{2}}{\rightarrow} \mathrm{X}_{2}+k_{5} \mathrm{CO}_{2}+k_{6} \mathrm{CH}_{4}
$$


where in the first step, acidogenic bacteria $\left(X_{1}\right)$ consume the organic substrate $\left(S_{1}\right)$ to generate VFA (mainly composed of acetate, propionate and butyrate) and $\mathrm{CO}_{2}$. In the second step, the produced VFA $\left(S_{2}\right)$ are consumed by the methanogenic bacteria $\left(\mathrm{X}_{2}\right)$ for growth and production of $\mathrm{CO}_{2}$ and methane.

Under some specific assumptions about the reactor $p H$ and temperature, Bernard et al. [6] proposed a suitable nonlinear mass balance model, named the AM2 model, incorporating the electrochemical equilibria. The later has been chosen in this work due to its ability of reflecting the reactor destabilisation when the VFA accumulation occurs. It includes 6 state variables whose dynamics are given by the following equations:

$$
\begin{aligned}
\dot{S}_{1}= & D\left(S_{1 i n}-S_{1}\right)-k_{1} \mu_{1}\left(S_{1}\right) X_{1} \\
\dot{X}_{1}= & \left(\mu_{1}\left(S_{1}\right)-\alpha D\right) X_{1} \\
\dot{S}_{2}= & D\left(S_{2 i n}-S_{2}\right)+k_{2} \mu_{1}\left(S_{1}\right) X_{1}-k_{3} \mu_{2}\left(S_{2}\right) X_{2} \\
\dot{X}_{2}= & \left(\mu_{2}\left(S_{2}\right)-\alpha D\right) X_{2} \\
\dot{Z}= & D\left(Z_{i n}-Z\right) \\
\dot{C}= & D\left(C_{i n}-C\right)-q_{c}(\xi)+k_{4} \mu_{1}\left(S_{1}\right) X_{1} \\
& +k_{5} \mu_{2}\left(S_{2}\right) X_{2}
\end{aligned}
$$

Herein, $S_{1}$ is the concentration of the organic matter to be digested, $X_{1}$ is the concentration of the acidogenic bacteria responsible for the degradation of organic matter, $S_{2}$ is the VFA concentration which is supposed to behave like pure acetate and $X_{2}$ is the methanogenic bacteria concentration. Then, $Z$ represents the alkalinity which is the sum of acetate and bicarbonate concentrations. $C$ is the inorganic carbon concentration in the digester (assumed to be the sum of the dissolved $\mathrm{CO}_{2}$ and bicarbonate) and $q_{c}$ is the $\mathrm{CO}_{2}$ gas flow rate. $S_{1 i n}$ and $S_{2 i n}$ are the inlet substrate and VFA concentrations, respectively, and $D$ is the dilution rate. $k_{i}$ represent the yield coefficients given in Table I and Table II at the end of the paper and $\alpha \in[0,1]$ reflects the digester heterogeneity: $\alpha=0$ for an ideal fixed bed reactor and $\alpha=1$ for an ideal Continuous Stirred Tank Reactor (CSTR). Finally, the acidogenic bacteria growth rate $\mu_{1}\left(S_{1}\right)$ depends on the concentration $S_{1}$ and is given by a Monod function:

$$
\mu_{1}\left(S_{1}\right)=\mu_{1 \max } \frac{S_{1}}{S_{1}+k_{s 1}}
$$

where $\mu_{1 \max }$ is the maximum bacterial growth rate and $k_{s 1}$ is the half saturation constant associated with the substrate $S_{1}$. However, the methanogenic bacteria growth rate $\mu_{2}\left(S_{2}\right)$ is modeled such that the methanogenic bacteria inhibition due to the VFA accumulation is taken into account. It is modeled by a Haldane function as the following:

$$
\mu_{2}\left(S_{2}\right)=\mu_{2 \max } \frac{S_{2}}{S_{2}+k_{s 2}+\frac{S_{2}^{2}}{k_{I 2}}}
$$

where $\mu_{2 \max }$ is the maximum bacterial growth rate without inhibition, $k_{s 2}$ and $k_{I 2}$ are the saturation and the inhibition constants, respectively, associated with the acetate concentration $S_{2}$.
Considering the low solubility of the methane, its concentration in the liquid phase is neglected and its flow rate in the gas phase is given by:

$$
Q_{M}=k_{6} \mu_{2}\left(S_{2}\right) X_{2}
$$

For further details of the model the reader is referred to [6]. Since our objective is the methane flow rate control, we exploit the cascaded structure of the model (3)-(9) by using only the reduced model (3)-(6) and (12) for the design of the control law.

\section{Methane Flow Rate Control}

Throughout this section, we suppose that we have no control on the incoming waste concentrations $S_{1 i n}$ and $S_{2 i n}$. Therefore, we act only on the dilution rate $D$ to control the methane flow rate in spite of the inlet concentration changes. To do so, we exploit the a priori knowledge of the system to control $Q_{M}$ by applying a linearized control as the following:

$$
\frac{d Q_{M}}{d t}-\lambda\left(Q_{M}^{*}-Q_{M}\right)=0
$$

where $Q_{M}^{*}$ is the desired methane flow rate and $\lambda$ is a design parameter which depends on the desired close loop performance. Now, using (12) the time derivative of $Q_{M}$ is given by:

$$
\frac{d Q_{M}}{d t}=k_{6} \frac{d \mu_{2}\left(S_{2}\right)}{d t} X_{2}+k_{6} \mu_{2}\left(S_{2}\right) \frac{d X_{2}}{d t}
$$

Thus, substituting (6) in (14) and using (12) we get the following relationship:

$$
\frac{d Q_{M}}{d t}=k_{6} \frac{d \mu_{2}\left(S_{2}\right)}{d t} X_{2}+Q_{M}\left(\mu_{2}\left(S_{2}\right)-\alpha D\right)
$$

Moreover, the dynamic of $\mu_{2}\left(S_{2}\right)$ can be derived as

$$
\frac{d \mu_{2}\left(S_{2}\right)}{d t}=\frac{d \mu_{2}\left(S_{2}\right)}{d S_{2}} \frac{d S_{2}}{d t}
$$

which can be further expressed using (5), (11) and (12) as

$$
\begin{aligned}
\frac{d \mu_{2}\left(S_{2}\right)}{d t}= & \frac{\mu_{2 \max }\left(k_{s 2} k_{I 2}-S_{2}^{2}\right)}{k_{I 2}\left(S_{2}+k_{s 2}+\frac{S_{2}^{2}}{k_{I 2}}\right)^{2}} . \\
& \cdot\left(D\left(S_{2 i n}-S_{2}\right)+k_{2} \mu_{1}\left(S_{1}\right) X_{1}-\frac{k_{3}}{k_{6}} Q_{M}\right)
\end{aligned}
$$

We equivalently rewrite (17) as follows:

$$
\begin{aligned}
\frac{d \mu_{2}\left(S_{2}\right)}{d t}= & \frac{k_{s 2} k_{I 2}-S_{2}^{2}}{k_{I 2} \mu_{2 \max }} \cdot \frac{\mu_{2}^{2}\left(S_{2}\right)}{S_{2}^{2}} . \\
& \cdot\left(D\left(S_{2 i n}-S_{2}\right)+k_{2} \mu_{1}\left(S_{1}\right) X_{1}-\frac{k_{3}}{k_{6}} Q_{M}\right)
\end{aligned}
$$

Now let $\theta$ be a variable defined as:

$$
\theta=\frac{k_{s 2} k_{I 2}-S_{2}^{2}}{k_{I 2} \mu_{2 \max }} \cdot \frac{\mu_{2}^{2}\left(S_{2}\right)}{S_{2}^{2}}
$$

Hence, substituting (19) in (18) and using (12), the dynamic of $Q_{M}$ given by (15) is equally given by:

$$
\begin{aligned}
\frac{d Q_{M}}{d t}=\theta & Q_{M}\left(D\left(S_{2 i n}-S_{2}\right)+k_{2} \mu_{1}\left(S_{1}\right) X_{1}-\frac{k_{3}}{k_{6}} Q_{M}\right) \\
& +Q_{M}\left(\mu_{2}\left(S_{2}\right)-\alpha D\right)
\end{aligned}
$$


Using (13), this is equivalent to

$$
\begin{aligned}
\frac{\lambda\left(Q_{M}^{*}-Q_{M}\right)}{\theta Q_{M}}= & \left(D\left(S_{2 i n}-S_{2}\right)+k_{2} \mu_{1}\left(S_{1}\right) X_{1}-\frac{k_{3}}{k_{6}} Q_{M}\right)+ \\
& +\frac{1}{\theta}\left(\mu_{2}\left(S_{2}\right)-\alpha D\right)
\end{aligned}
$$

Thus, the dilution rate finally yields

$$
\begin{aligned}
D= & \left(\frac{1}{S_{2 i n}-S_{2}-\frac{\alpha}{\theta}}\right) \cdot \\
& \cdot\left(\frac{\lambda\left(Q_{M}^{*}-Q_{M}\right)}{\theta Q_{M}}-k_{2} \mu_{1}\left(S_{1}\right) X_{1}+\frac{k_{3}}{k_{6}} Q_{M}-\frac{\mu_{2}\left(S_{2}\right)}{\theta}\right)
\end{aligned}
$$

We can note that the control (22) does not require the measurement of $S_{1 \text { in }}$ and that all variables are known except the reaction rate $\mu_{1}\left(S_{1}\right) X_{1}$ which is unknown and must be estimated.

\section{Acetate Concentration Control}

The accumulation of VFA causes a $p H$ breakdown in the rector and consequently inhibits the methanogenesis. Actually, the methanogenic bacteria growth rate depends on the $S_{2}$ concentration and is of type Haldane which is a non-monotonic function, i.e:

$$
\left\{\begin{array}{llll}
\mu_{2}(0) & =\mu_{2}(\infty) & =0 & \\
\frac{d \mu_{2}\left(S_{2}\right)}{d S_{2}}>0 & \text { if } & 0 \leq S_{2} \leq S_{2}^{M} \\
\mu_{2}\left(S_{2}\right) & =\mu_{2}^{M} & \text { if } & S_{2}=S_{2}^{M} \\
\frac{d \mu_{2}\left(S_{2}\right)}{d S_{2}} & <0 & \text { if } & S_{2}>S_{2}^{M}
\end{array}\right.
$$

with $S_{2}^{M}=\sqrt{k_{I 2} \cdot k_{s 2}}$. Therefore, depending on the inputs of the system different steady states can be reached by the latter (for sake of brevity they will not be presented here, a detailed study of the possible equilibriums can be found in [4] and [10]).

Thus, to operate the process around a useful working equilibrium, $S_{2}$ should not exceed $S_{2}^{M}$. Moreover, in real operation the digesters are vulnerable to a shock load when the treated waste materials are coming from different origins. Thus, based on an a priori knowledge of the system the operator fixes a desired value $S_{2}^{*}$ for the $S_{2}$ concentration which is a compromise between two issues: safety of the digester and maximisation of the growth rate. Therefore, the objective herein is to bring $S_{2}$ towards $S_{2}^{*}$ regardless of the input concentrations.

In the following, we assume a discrete-time formulation, i.e. the variables are considered at discrete time steps $k \cdot \Delta T$ with the sampling period $\Delta T$. Mathematically speaking, we want that the error $e(k)=S_{2}(k)-S_{2}^{*}$ decreases exponentially from time instant $k$ to instant $k+1$, i.e:

$$
S_{2}(k+1)-S_{2}^{*}=\beta\left(S_{2}(k)-S_{2}^{*}\right)
$$

with $0 \leq \beta \leq 1$. By choosing $\beta=0$, we get:

$$
S_{2}(k+1)=S_{2}^{*}
$$

Now, using the first order Euler approximation, (5) can be formulated in a discrete-time version with the sampling period $\Delta T$ :

$$
\begin{aligned}
S_{2}(k+1)= & S_{2}(k)+\Delta T \cdot\left(D(k)\left(S_{2 i n}(k)-S_{2}(k)\right)+\right. \\
& \left.+k_{2} \mu_{1}\left(S_{1}(k)\right) X_{1}(k)-k_{3} \mu_{2}\left(S_{2}(k)\right) X_{2}(k)\right)
\end{aligned}
$$

Using (25), (26) and (12) we obtain:

$D(k)=\frac{S_{2}^{*}-S_{2}(k)+\Delta T\left(k_{2} \mu_{1}\left(S_{1}(k)\right) X_{1}(k)-\frac{k_{3}}{k_{6}} Q_{M}(k)\right)}{\Delta T\left(S_{2 i n}(k)-S_{2}(k)\right)}$

In (27) all variables are known except the reaction rate $\mu_{1}\left(S_{1}(k)\right) X_{1}(k)$ which is unknown and must be estimated.

\section{OBSERVER BASED ESTIMATOR FOR THE ACIDOGENESIS REACTION RATE}

First, let's denote by $\xi^{T}=\left[S_{1}, X_{1}, S_{2}, X_{2}\right]$ ( ${ }^{T}$ represents the transpose operator) the state vector of the model (3)-(6).

In real operation, the full vector of state variables is not measurable and only few information about the system are accessible. Moreover, the measurements of the bacteria concentrations are expensive and thus not very desirable from an industrial perspective. In addition, the reaction rates are not completely known. Therefore we assume that for the known inputs ( $D$ and $S_{2 i n}$ ) and known yield coefficients the only available online measurements are $S_{2}$ and $Q_{M}$ and we will design an observer-based estimator for the acidogenesis reaction rate:

$$
r_{1}=\mu_{1}\left(S_{1}\right) X_{1}
$$

Now, before describing the observer-based estimator, we remember that the dilution rate which is the inverse of the hydraulic retention time must be bounded $\left(0 \leq D<D_{\max }\right)$ in order to avoid the wash out of bacteria. Moreover, for bounded inputs of the system $\left(S_{1 i n}, S_{2 i n}\right)$ and zero or positive initial conditions of $\xi$, the positiveness and boundedness of the state variables have already been proven in [9] and [10]. Thus, we obtain:

$$
\begin{cases}\mu_{1}(0) & =0 \\ \frac{d \mu_{1}\left(S_{1}\right)}{d S_{1}} & >0 \quad \text { for } \quad S_{1} \geq 0 \\ \mu_{1}(\infty) & =m_{1}\end{cases}
$$

The design of the observer-based estimator for the acidogenesis will now be considered in the following. From (5), (12) and (28), we rewrite the dynamics of the acetate concentration as

$$
\dot{S}_{2}=D\left(S_{2 i n}-S_{2}\right)+k_{2} r_{1}-\frac{k_{3}}{k_{6}} Q_{M}
$$

from which the variable $r_{1}$ will be estimated using the following observer-based estimator:

$$
\begin{aligned}
& \dot{\hat{S}}_{2}=D\left(S_{2 i n}-S_{2 m}\right)+k_{2} \hat{r}_{1}-\frac{k_{3}}{k_{6}} Q_{M m}+\omega\left(S_{2 m}-\hat{S}_{2}\right) \\
& \dot{\hat{r}}_{1}=\delta\left(S_{2 m}-\hat{S}_{2}\right)
\end{aligned}
$$


where $S_{2 m}$ and $Q_{M m}$ are the measurements of $S_{2}$ and $Q_{M}$, respectively, subject to bounded noise $\epsilon_{1}$ and $\epsilon_{2}$ :

$$
\begin{aligned}
& S_{2 m}=S_{2}+\epsilon_{1} \\
& Q_{M m}=Q_{M}+\epsilon_{2}
\end{aligned}
$$

where $\omega$ and $\delta$ are the parameters of the observer-based estimator. To tune these parameters we consider the dynamics of the estimation errors $\left(\widetilde{S}_{2}=S_{2}-\hat{S}_{2}\right.$ and $\left.\widetilde{r}_{1}=r_{1}-\hat{r}_{1}\right)$ given by:

$$
\begin{aligned}
& \dot{\widetilde{S}}_{2}=-\omega \widetilde{S}_{2}+k_{2} \widetilde{r}_{1}+(D-\omega) \epsilon_{2}+\frac{k_{3}}{k_{6}} \epsilon_{1} \\
& \dot{\widetilde{r}}_{1}=-\delta \widetilde{S}_{2}+\dot{r}_{1}-\omega \epsilon_{2}
\end{aligned}
$$

Defining $\widetilde{X}^{T}=\left[\widetilde{S}_{2}, \widetilde{r}_{1}\right]$, we rewrite (33) in matrix format as follows:

$$
\begin{gathered}
\dot{\tilde{X}}=A X+u \\
{\left[\begin{array}{c}
\dot{\widetilde{S}}_{2} \\
\dot{\tilde{r}}_{1}
\end{array}\right]=\left[\begin{array}{cc}
-\omega & k_{2} \\
-\delta & 0
\end{array}\right]\left[\begin{array}{l}
\widetilde{S}_{2} \\
\widetilde{r}_{1}
\end{array}\right]+\left[\begin{array}{l}
(D-\omega) \epsilon_{2}+\frac{k_{3}}{k_{6}} \epsilon_{1} \\
\dot{r}_{1}-\delta \epsilon_{2}
\end{array}\right]}
\end{gathered}
$$

As a consequence of (29) and the boundedness of both $D$ and the noise measurements the input $u$ of the error system (33) is bounded. Furthermore it is a classical result in observers theory that the observer-based estimator (31) is stable and the estimation errors of the system (33) are bounded if the matrix $A$ is stable (see e.g. [9] for more details). Therefore, the parameters $\omega$ and $\delta$ are designed in a way that the matrix $A$ remains stable. For a detailed methodology of how to tune $\omega$ and $\delta$ the reader is referred to [9].

\section{AdAptive Control For the AnAerobic Digestion PROCESS}

The main objective of this work is to control the methane flow rate such that the biogas plant satisfies a requested energy demand. As discussed however, due to the reactor acidification risk the plant can only respond to the demand if its safety is not endangered. Thus, combining the control laws given by (22) and (27) and the estimations delivered by the observerbased estimator (31) the full adaptive control algorithm of the process is given by:

$$
D= \begin{cases}\text { Eq. (22) } & \text { if } S_{2}(k+1) \leq S_{2}^{M} \\ \text { Eq. (27) } & \text { else }\end{cases}
$$

where the term $\mu_{1}\left(S_{1}(k)\right) X_{1}(k)$ in both (22) and (27) is replaced by its estimate $\widehat{r}_{1}$ generated by the observer-based estimator (31).

\section{Simulation Results}

In order to validate the proposed overall control algorithm as described in Section VI with the help of suitable simulations, the used input concentrations $\left(S_{1 i n}, S_{2 i n}\right)$ applied in the simulations have been chosen to vary in a sufficiently wide range of expected operating conditions including the shock loading case, see Figs. (1) and (2). Moreover, we added white noise of different amplitudes to the measurements of $S_{2}$ and $Q_{M}$. Concerning the reactor heterogeneity it is neither a perfect CSTR nor a perfect fixed bed digester $(\alpha=0.5)$.

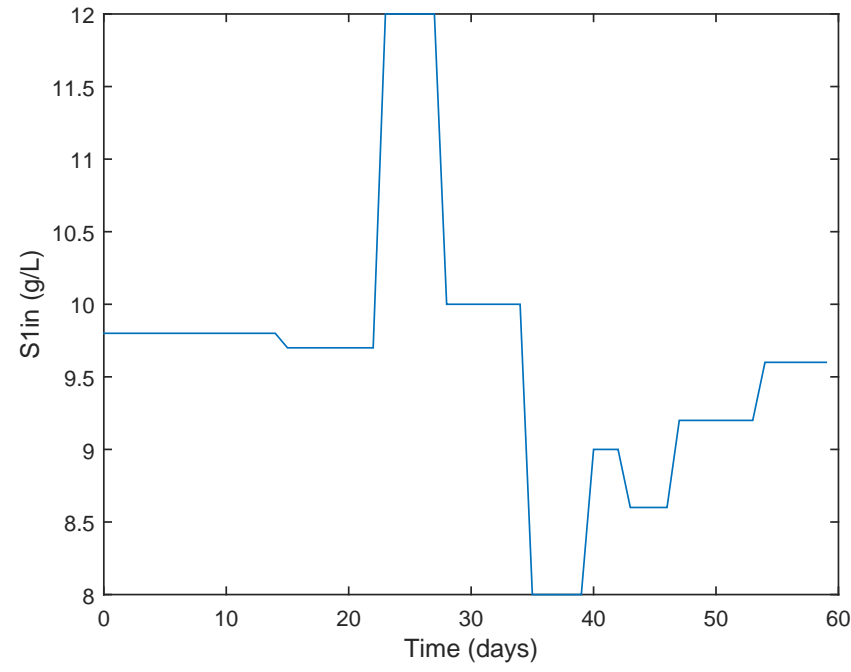

Fig. 1. Inlet substrate concentration $\left(S_{1 i n}\right)$.

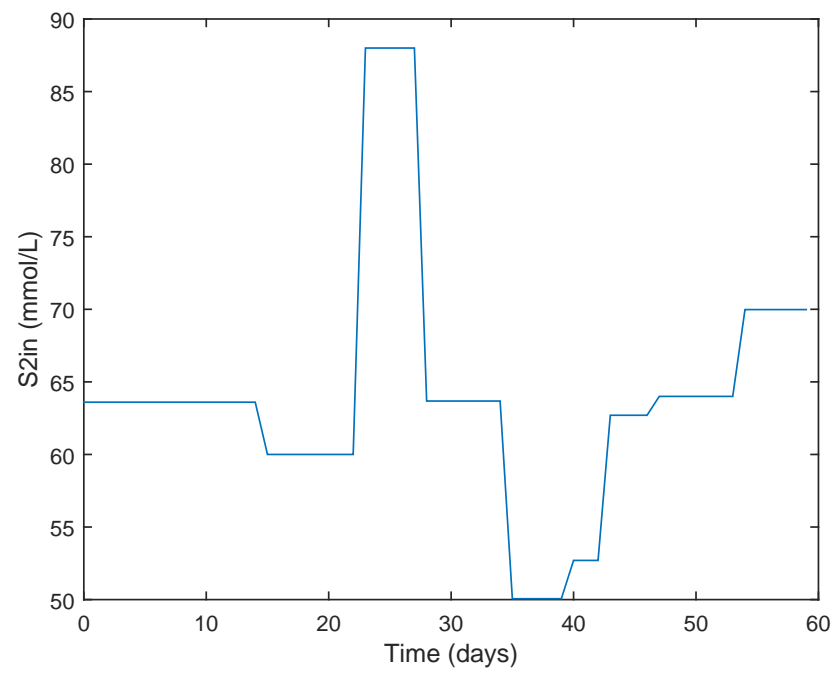

Fig. 2. Inlet volatile fatty acids concentration $\left(S_{2 i n}\right)$.

Regarding the design of the parameters of the observer-based estimator, they were calculated such that the matrix $A$ in (34) has distinct eigenvalues $(-5,-6)$. Finally, according to the desired performance the parameter $\lambda$ is set equal to 0.4 .

In Fig. (6) we compare the methane flow rate for the uncontrolled process and for the case where the designed overall control algorithm is applied. We can conclude that the system follows the reference in spite of the large input changes while avoiding the VFA accumulation and the bacteria washout, see the results depicted in Figs. (3, 4 and 5).

Similarly, the results shown in Fig. (7) underline that we obtain a very good estimation of the acidogenesis reaction rate although we applied the noisy measurements. 


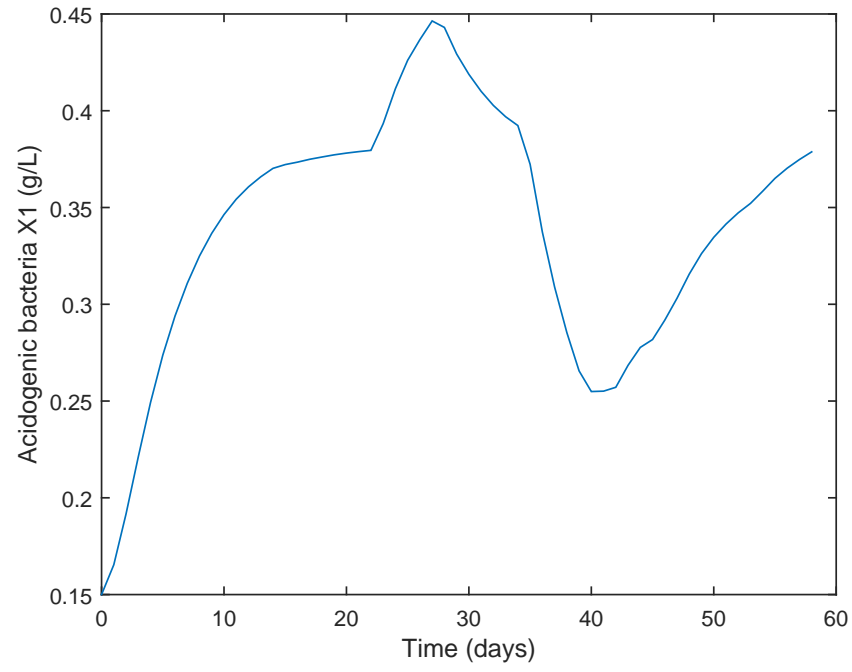

Fig. 3. Acidogenic bacteria concentration in the reactor $\left(X_{1}\right)$.

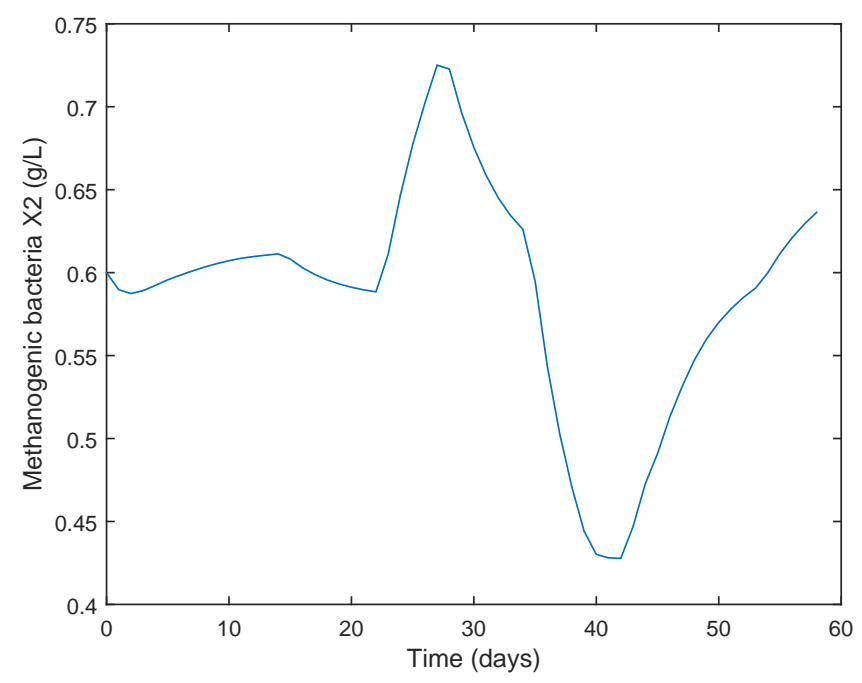

Fig. 4. Methanogenic bacteria concentration in the reactor $\left(X_{2}\right)$.

\section{ViII. Conclusion And Perspectives}

In order to integrate biogas plants in a virtual power plant and to satisfy a requested energy demand, we proposed a suitable control algorithm to control the methane flow rate of the AD process of the biogas plant. The designed control algorithm also preserves the safety of the digester by majoring the dilution rate value according to the VFA concentration. Moreover, we propose an observer-based estimator for the estimation of the acidogenesis reaction rate in order to avoid expensive and complex measurements of the bacteria concentrations. Finally, we provided some simulation results to validate the proposed algorithm also under large changes of the input concentrations. These first simulation results are promising and underline the applicability of the proposed approach. Consequently, we target to extend the designed

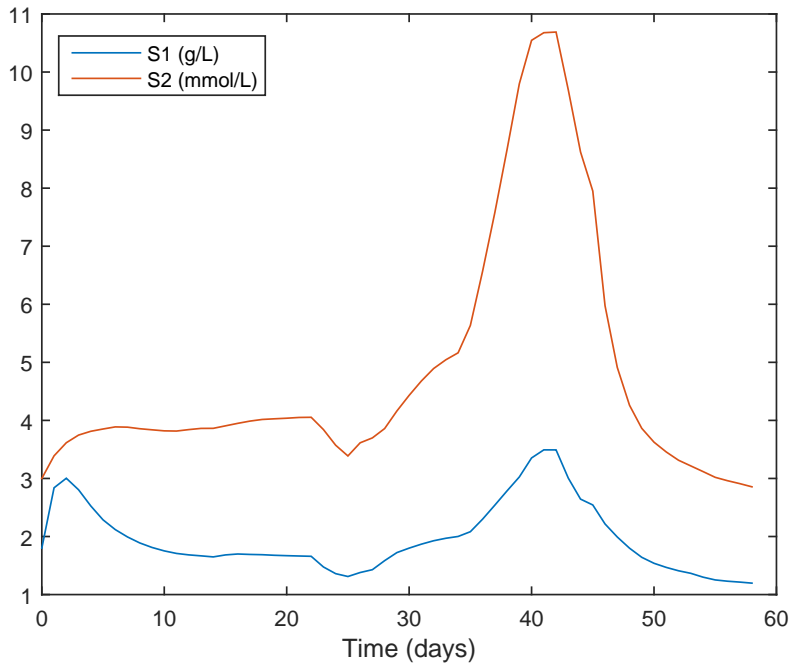

Fig. 5. Organic substrate and VFA concentrations in the reactor.

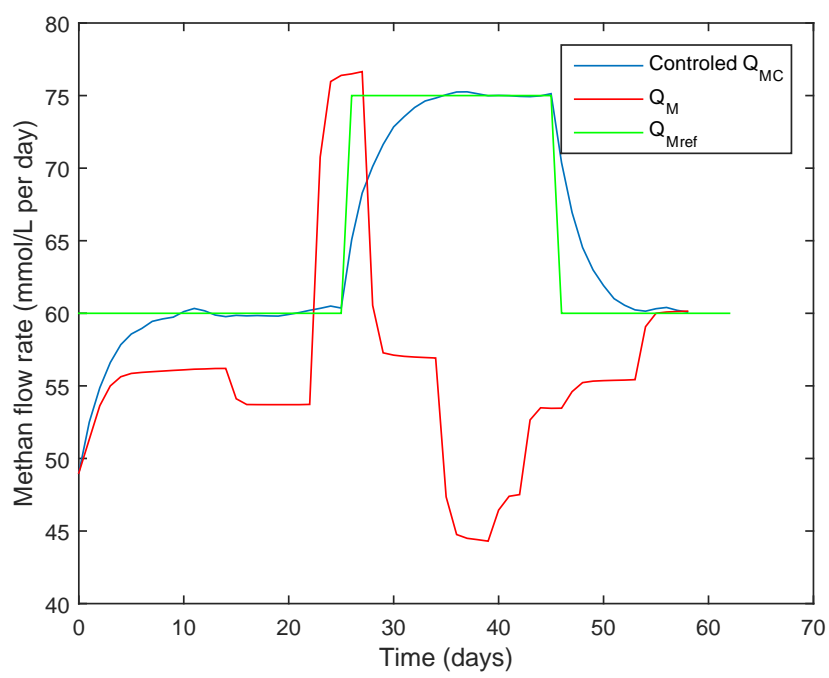

Fig. 6. Comparison of the controlled and uncontrolled $Q_{M}$

algorithm in a way that also the measurement's delays will be taken into account. Finally, we will also investigate in our future work how to control the quantity and quality of the biogas.

\section{REFERENCES}

[1] F. Prause, 1997. Mathematical modeling of the anaerobic treatment process representation of previous approaches and applications of a selected model using the example of the KA Marne. $\mathrm{PhD}$ thesis, Institute of Urban Water Management and Waste Technology, University of Hannover, Germany, 1997.

[2] S. Weinrich, M. Nelles, R.F. Jacobi, E. Mauky 2013. Praxisnahe Modellierung von Biogasanlagen Vergleich unterschiedlicher Modellanstze. Ifak Workshop in Leipzig.

[3] D.J. Batstone, J. Keller, I. Angelidaki, S.V. Kalyuzhnyi, S.G. Pavlostathis, A. Rozzi, W.T.M. Sanders, H. Siegrist, V.A. Vavilin 2002. Anaerobic Digestion Model No. 1. IWA Publishing, IWA STR No. 13. 


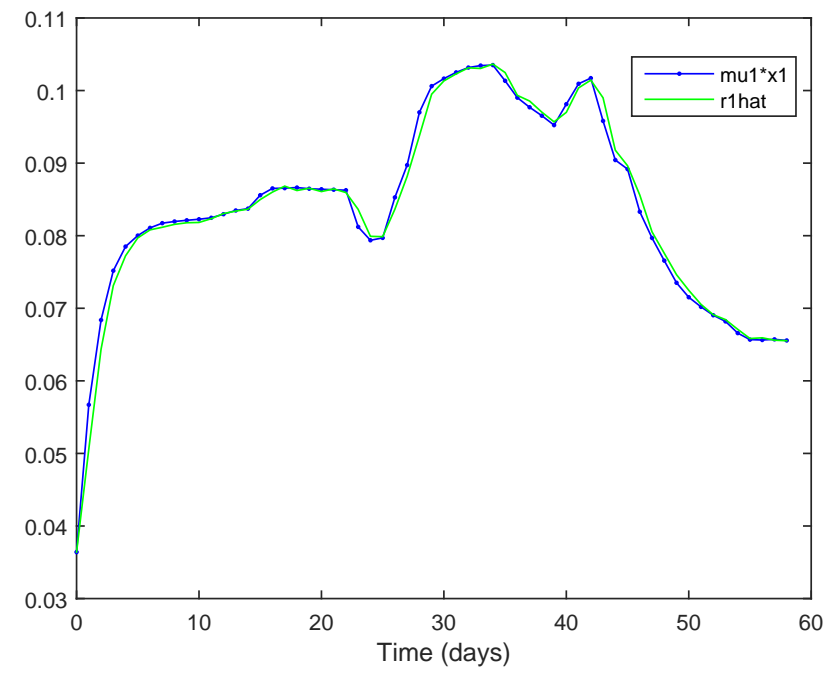

Fig. 7. Acidogenesis reaction rate estimation.

TABLE I

NOMENCLATURE

\begin{tabular}{ll}
\hline \hline Acronyms & Definition \\
\hline$k_{1}$ & yield coeff. for substrate degradation \\
$k_{2}$ & yield coeff. for VFA production \\
$k_{3}$ & yield coeff. for VFA consumption \\
$k_{4}$ & yield coeff. for $C O_{2}$ production \\
$k_{5}$ & yield coeff. for $C O_{2}$ production \\
$k_{6}$ & yield coeff. for $C h_{4}$ production \\
$\mu_{1 \max }$ & Maximum acidogenic bacteria growth rate \\
$\mu_{2 \max }$ & Maximum methanogenic bacteria growth rate \\
$k_{s 1}$ & Half saturation constant associated with $S_{1}$ \\
$k_{s 2}$ & Half saturation constant associated with $S_{2}$ \\
$k_{I 2}$ & Inhibition constant associated with $S_{2}$ \\
\hline \hline
\end{tabular}

TABLE II

NOMENCLATURE-CONTINUATION

\begin{tabular}{lll}
\hline \hline Acronyms & Units & Values \\
\hline$k_{1}$ & $g \cdot g^{-1}$ of acidogenic bacteria & 42.14 \\
$k_{2}$ & mmole. $g^{-1}$ of acidogenic bacteria & 116.5 \\
$k_{3}$ & mmole. $g^{-1}$ of methanogenic bacteria & 268 \\
$k_{4}$ & mmole. $g^{-1}$ & 454 \\
$k_{5}$ & mmole. $g^{-1}$ & \\
$k_{6}$ & mmole. $g^{-1}$ & \\
$\mu_{1 \max }$ & day & 1.2 \\
$\mu_{2 \max }$ & day & 0.74 \\
$k_{s 1}$ & g.l & 7.1 \\
$k_{s 2}$ & mmol. $l^{-1}$ & 9.28 \\
$k_{I 2}$ & mmol.l & 256 \\
\hline \hline
\end{tabular}

[4] J. Hess, O. Bernard, J. P. Steyer 2008. Thse: Modlisation de la Qualit du Biogas Produit par un Fermenteur Mthanogne et sa Stratgie de Rgulation en Vue de sa Valorisation. Universit de Nice - Sophia Antipolis.

[5] P. Renard, V. Van Breusegem, M.T Nguyen, E.J Nyns 1991. Implementation of an adaptive Controller for the Startup and Steady-State Running of a Biomethanation Process Operated in the CSTR Mode. Biotechnology and Bioengineering, Vol. 38, P.805-812.

[6] O. Bernard, Z. Hadj-Sadok, D. Dochain, A. Genovesi and J. Steyer 2001. Dynamical Model Development and Parameter Identification for an Anaerobic Wastewater Treatment Process. Biotechnology and Bioengi- neering, Vol. 75, No.4.

[7] S. Marsili-Libelli, S. Beni 1994. Shock load modelling in the anaerobic digestion process. Ecological Modelling 84 (1996) 215-232.

[8] J. Hess,O. Bernard 2006. Mechanistic kLa Modelling in an Up-flow Anaerobic Digester. Proceedings of the 5th Symposium on Mathematical Modeling, Vienna, Austria, MATHMOD.

[9] G. Bastin, D. Dochain 1990. On-line Estimation and Adaptive Control of Bioreactors. Elsevier Science Publishers, Amsterdam and New York.

[10] B. Benyahia 2012. Thse: Modlisation et Observation des Bioprocds Membranes: Application la Digestion Anarobie. Universit de Tlemcen, Algrie et Univesit de Montpelier 2, France. 